is naturaleza 


\section{NATURALEZA Y LIBERTAD}

Revista de estudios interdisciplinares

Número 1

Málaga, 2012

Esta revista es accesible on-line en el siguiente portal: http://grupo.us.es/naturalezayl/ 


\section{Naturaleza y Libertad}

Revista de estudios interdisciplinares

Número 1

Directores: Juan Arana, Universidad de Sevilla; Juan José Padial, Universidad de Málaga; Francisco Rodríguez Valls, Universidad de Sevilla.

Secretaria: Avelina Cecilia Lafuente, Universidad de Sevilla.

Consejo de Redacción: José Luis González Quirós, Universidad Juan Carlos I, Madrid; Francisco Soler, Universität Dortmunt / Universidad de Sevilla; Pedro Jesús Teruel. Universidad CEU Cardenal Herrera; Héctor Velázquez, Universidad Panamericana, México.

Consejo Editorial: Mariano Alvarez, Real Academia de Ciencia Morales y Políticas; Allan Franklin, University of Colorado; Michael Heller, Universidad Pontificia de Cracovia; Manfred Stöcker, Universität Bremen; William Stoeger, University of Arizona.

Consejo Asesor: Rafael Andrés Alemañ Berenguer. Universidad M. Hernández de Elche; Juan Ramón Álvarez, Universidad de León; Luciano Espinosa, Universidad de Salamanca; Miguel Espinoza, Université de Strasbourg; José Manuel Giménez Amaya, Universidad de Navarra; Karim Gherab Martín, Urbana University, Illinois; Martín López Corredoira, Instituto Astrofísico de Canarias; Alfredo Marcos, Universidad de Valladolid; Javier Monserrat, Universidad Autónoma de Madrid; Leopoldo Prieto, Colegio Mayor San Pablo, Madrid; Ana Rioja, Universidad Complutense, Madrid. Madrid; José Luis Rodríguez Recio, Universidad Complutense, Madrid; Javier Serrano, TEC Monterrey (México); Hugo Viciana, Université

Paris I; Claudia Vanney, Universidad Austral, Buenos Aires; José Domingo Vilaplana, Huelva.

\section{Redacción y Secretaría:}

Naturaleza y Libertad. Revista de estudios interdisciplinares. Departamento de Filosofía y Lógica. Calle Camilo José Cela s.n. E-41018 Sevilla.

Depósito Legal: MA2112-2012

๔ 954.55.77.57 Fax: 954.55.16.78. E-mail: jarana@us.es

๑ Naturaleza y Libertad. Revista de Filosofía, 2012 


\section{ÍNDICE}

MANIFIESTO

\section{ESTUDIOS}

Rafael Andrés Alemañ Berenguer. Universidad Miguel Hernández de Elche

La relación mente-materia y el monismo neutral.

Fernando García-Cano Lizcano. Ciudad Real

Evolucionismo y utopia de género

Miguel Ángel Herrero. Universidad Politécnica de Madrid

Instrumentalismo y realismo en la física de James C. Maxwell...

Juan Miguel Suay Belenguer. UNED

La mente mecánica.

José Luis Yepes Hita. Universidad de Murcia

Los «Anales de Física» de L.W. Gilbert.

DisCUSIONES

Francisco José Soler Gil. Universidad de Sevilla - T. Universität Dortmund

¿Se puede naturalizar la epistemología?

Antonio Diéguez. Universidad de Málaga

La opción naturalista. Una respuesta a Francisco Soler

Francisco José Soler Gil. Universidad de Sevilla - T. Universität Dortmund

¿Qué menos que un milagro podría salvar la epistemología naturalista? .265

Antonio Diéguez. Universidad de Málaga

Filosofía sin milagros. Comentarios finales a la contrarréplica de Francisco Soler ...273

CRÍTICA DE LIBROS

Pedro Jesús Teruel, Filosofia y ciencia en Hipatia, (Ángel Martínez Sánchez) . 285 Noticias y COMENTARIOS

Miguel Palomo, Universidad de Sevilla

Si no hay cuerpo, no hay mente: una discusión sobre consciencia y libertad. 


\title{
Instrumentalismo y realismo en la física de James C. Maxwell
}

\author{
Miguel Ángel Herrero \\ Universidad Politécnica de Madrid
}

\begin{abstract}
Resumen: Los tres artículos publicados entre 1856 y 1864 por Maxwell han sido analizados con el fin de estudiar el método de la analogía científica utilizado en la construcción de la teoría del campo electromagnético. Dicha teoría consiste en un formalismo matemático con significado físico. El procedimiento seguido por Maxwell proporciona un medio idóneo para examinar cómo se construyen los términos científicos y cuál es el papel que desempeñan en la formación de la teoría científica. A tenor del análisis realizado, la teoría de Maxwell muestra que el papel de la ciencia no es revelar entidades reales escondidas bajo apariencias sensibles, sino construir un formalismo matemático significante a partir de datos experimentales.
\end{abstract}

Palabras clave: Método científico, instrumentalismo, realismo, campo electromagnético, Maxwell.

Abstract: The three papers published between 1856 and 1864 by Maxwell have been analyzed in order to study the method of scientific analogy applied to construct the theory of electromagnetic field. The theory consists of a mathematical formalism with physical meaning. The procedure followed by Maxell is a valuable subject to examine in order to understand how scientific terms are defined and it is also a useful way of investigating the function they played in the construction of theories. This analysis shows that the role of the empirical science is not to discover real entities hidden under sensible appearances, but to build up a meaningful mathematical formalism from experimental data.

Key words: Scientific method, instrumentalism, realism, electromagnetic field, Maxwell.

Recibido: 26/07/2011. Aprobado: 10/12/2011. 
Naturaleza y Libertad. Revista de estudios interdisciplinares. Número 1, 2012. ISSN: 2254-9668

\section{INTRODUCCIÓN}

En la historia de la investigación científica, James Clerk Maxwell ocupa un lugar destacado por sus trabajos sobre diversos fenómenos físicos. En termodinámica sentó las bases de la física estadística con la teoría cinética de gases, en electromagnetismo obtuvo una formulación teórica que abarca a todos los fenómenos entonces observados y que se extiende a la propagación de la luz. Su legado intelectual fue doble, por un lado, estrictamente científico, en el que sobresale la teoría electromagnética y, por otro, metodológico, por su aportación al concepto físico-matemático de campo electromagnético, fundamento de teorías posteriores. Así lo atestigua el siguiente comentario de Einstein, con ocasión del centenario del nacimiento del físico escocés.

[...] si dejamos a un lado los importantes resultados individuales que produjo la investigación de Maxwell a lo largo de su vida en concretos ámbitos de la física y centramos nuestra atención en los cambios que él produjo en nuestra concepción de la naturaleza de la realidad física, podemos decir que antes de Maxwell la realidad física, en la medida en que era la representación de los procesos naturales, consistía en partículas materiales, cuyas variaciones consisten solamente en movimientos regidos por ecuaciones en derivadas parciales. Desde los tiempos de Maxwell, la realidad física se considera representada por campos continuos, regidos por ecuaciones en derivadas parciales y no susceptibles de interpretación mecánica. Este 
Naturaleza y Libertad. Revista de estudios interdisciplinares. Número 1, 2012. ISSN: 2254-9668

cambio en la concepción de la realidad es el más profundo y el más fecundo que la física ha experimentado desde el tiempo de Newton ${ }^{1}$ [Cursiva añadida].

La posición realista de Einstein que hemos destacado en la referencia anterior es bien conocida. Según el científico alemán, la ciencia describe la realidad física del modo más fiel posible. Para lo cual, utiliza representaciones tales como la del campo electromagnético, expresadas mediante el lenguaje matemático. En este último aspecto la descripción de los fenómenos electromagnéticos difiere de las teorías mecánicas, pues éstas se basan en el concepto de partícula material. Por tanto, la actividad científica supone partir de una realidad física preexistente, que admite ser representada y descrita en lenguaje matemático. Sin embargo, esta concepción realista no implica que dicha representación sea una copia fidedigna de la realidad, sino que debe ser construida por la razón para dar cuenta de los hechos observados. De acuerdo con este planteamiento, sería posible conocer el mundo natural a través de tales representaciones o modelos científicos. A partir de aquí, la filosofía de la ciencia se ocuparía en señalar hasta qué punto la ciencia es capaz de conocer la realidad de los fenómenos naturales que se observan y cómo se pueden describir mediante las teorías científicas.

En el lado opuesto del enfoque realista se halla la posición instrumentalista, cuyas características fueron descritas por Popper a partir de

1 Einstein, A., "Maxwell's influence on the development of the conception of physical reality". En: James Clerk Maxwell. A Commemoration volume 1831-1931. Cambridge, University Press, 1931: 66-73. 
la concepción de Berkeley, quien podría considerarse un instrumentalista avant la lettre. Según esta posición filosófica, "las teorías científicas no son más que instrumentos para el cálculo y la predicción de los fenómenos inminentes. No describen el mundo ni aspecto alguno de él. No pueden hacerlo porque están completamente faltas de sentido" ${ }^{2}$. De este modo, el enfoque instrumentalista adjudica a las teorías científicas el papel de meras herramientas de cálculo, sin ningún trasfondo interpretativo posible que las vincule a la realidad física. Con ello, la ciencia cumple con la mera función de construir artefactos matemáticos con una exclusiva finalidad práctica, tales como hacer predicciones y desarrollar aplicaciones técnicas.

Ambas concepciones no son del todo antagónicas pues comparten la idea de una ciencia como construcción racional, realizada a partir de los datos observados. Su diferencia consiste en que, el realismo atribuye una capacidad cognoscitiva a las teorías científicas, mientras que el instrumentalismo se la niega. Para éste, las teorías científicas no son capaces de dar a conocer las entidades que forman el mundo empírico, ni pueden expresar las interacciones que se producen entre ellas. Por el contrario, el realismo admite que las teorías científicas proporcionan algún tipo de conocimiento acerca de las entidades naturales y de sus operaciones.

2 Popper, K., Realismo y el objetivo de la ciencia". Post Scriptum a La lógica de la investigación cientifica. 1998, vol. 1: 147 [Cursiva en el original]. 
A lo largo de las páginas que siguen, nos proponemos analizar estos dos planteamientos, para lo cual, examinaremos el método seguido en la construcción de la teoría del campo electromagnético. Esta teoría, por la amplitud de su contenido, ha servido de fundamento metodológico a otras teorías físicas. Para Einstein "estaba justificado creer que por el camino iniciado con tanto éxito por Faraday y Maxwell se iría encontrando poco a poco una base firme para toda la física"3; como de hecho así ocurrió. Por esto, aunque nuestro análisis se refiere a un caso concreto, las conclusiones no se circunscriben a él, sino que, pueden ser aplicadas a la ciencia, en general y en mayor grado a la física.

Para abordar esta tarea ha sido preciso adoptar una doble perspectiva: científica y filosófica. En cuanto a la primera, hay que señalar que el análisis científico del método empleado por Maxwell nos obliga a describir algunos aspectos técnicos, los cuales pueden abordarse sin necesidad de descender a desarrollos matemáticos, utilizando el texto original en el que se expone con detalle el método seguido. Al hacerlo así, ponemos en práctica la recomendación bien experimentada de Einstein: "si alguien quisiera averiguar algo acerca de los métodos que los físicos utilizan, tendrá que seguir al pie de la letra este consejo: no escuchar sus palabras, sino prestar total atención a lo que los investigadores hagan" ${ }^{4}$.

3 Einstein, A., Notas autobiográficas, Madrid, Alianza Editorial, 1983: 39.

4 Einstein, A., Mis ideas y opiniones, Barcelona, Bon Ton, 2000: 242. 
El punto de partida señalado nos parece el más adecuado, puesto que las cuestiones planteadas sobre la naturaleza de las teorías científicas no pueden encontrar una respuesta satisfactoria exclusivamente en el plano especulativo, ante todo, es preciso descender al plano científico. Por consiguiente, hemos de situarnos en un marco interdisciplinar, delimitado por las tres materias que intervienen, esto es, la ciencia experimental, historia de la ciencia y filosofía de la ciencia.

\section{LA BASE EMPÍRICA DE LA TEORÍA}

El propósito, claramente expresado por Maxwell en On Faraday lines of force, es construir una teoría que dé unidad y coherencia a las diversas explicaciones que existían sobre los fenómenos eléctricos y magnéticos ya conocidos: "[se trata pues de una tarea de] simplificación y de reducción de los resultados obtenidos en investigaciones previas, de tal forma que la mente pueda comprenderlos" ${ }^{\text {. }}$.

¿Cuáles eran esos resultados procedentes de investigaciones previas? En 1855 la ciencia sobre los fenómenos eléctricos y magnéticos era ante todo experimental. En algunos casos, existían teorías que daban explicaciones relativas a un conjunto de hechos empíricos, mientras que en otros casos, los resultados experimentales no encon-

5 Maxwell, J. C., The Scientific Papers of James Clerk Maxwell, Dover Publications, 2003, vol. 1: 155 . 
traban una explicación teórica convincente. Existían formulaciones matemáticas que se referían a la trasmisión de la electricidad a través de hilos conductores y a la fuerza de atracción entre corrientes, pero, eran explicaciones aisladas del resto, sin relación con las que servían para explicar otros fenómenos eléctricos.

Los fenómenos empíricos de origen eléctrico conocidos en el tiempo de Faraday y Maxwell pueden resumirse del siguiente modo. En primer lugar, los debidos a la electricidad estática, que producían efectos mensurables de atracción y repulsión entre cuerpos dotados de carga eléctrica. En segundo lugar, los efectos eléctricos relacionados con el movimiento de cargas en conductores, como la conducción eléctrica (o "conducción galvánica") y la atracción o repulsión de conductores. Con relación a los fenómenos originados por las propiedades magnéticas de algunos minerales, eran conocidos los efectos de atracción y repulsión entre barras magnéticas o imanes, similares a los producidos por la electricidad estática, asimismo, se podía detectar el efecto magnético producido por una corriente eléctrica. Este último hallazgo era especialmente relevante puesto que expresaba por vía experimental la relación entre dos fenómenos en principio diferentes, la electricidad y el magnetismo. Igualmente, eran detectables los efectos dinámicos del magnetismo sobre cargas en movimiento (en el espacio libre, o en un conductor). Finalmente, se conocían las acciones a través del espacio, es decir, sin materia interpuesta, bien sea, entre cuerpos eléctricos o entre cuerpos magnéticos. Este tipo de interacción fue 
denominada inducción por Faraday, referida tanto a la inducción eléctrica, como a la magnética.

En consecuencia, los fenómenos eléctricos y magnéticos pueden ser clasificados en tres categorías. La primera, en relación con la generación de tales fenómenos, los cuales pueden tener un origen natural o artificial, cuando se producen utilizando máquinas, cuya energía, a su vez, puede almacenarse en baterías, en virtud de procesos químicos. La segunda categoría se refiere a la transmisión de los efectos eléctricos que se propagan a través de materiales conductores o se trasmiten sin necesidad de un soporte material. Por ejemplo, la atracción o repulsión de una pieza de hierro situada en el espacio a cierta distancia de una barra de material magnético y análogamente cuando se trata de interacciones producidas entre cuerpos electrizados en el espacio. La tercera categoría es la interacción mutua (descubierta por Oersted en 1819) entre la electricidad y el magnetismo, cuando una corriente eléctrica produce efectos magnéticos y - en sentido contrario- cuando el magnetismo produce efectos eléctricos.

En resumen, este es el conjunto de hechos observados que forma la base empírica de la teoría electromagnética. Ahora bien, la descripción teórica y formulación de tales hechos y de los sucesos empíricos en general implica situarlos dentro de un marco conceptual coherente. A diferencia de la observación común, la observación científica se realiza con una finalidad determinada, es decir, está guiada por un objetivo científico, lo cual implica llevar a cabo determinadas operaciones experimentales. Tales trabajos de laboratorio se realizan siempre en unas 
circunstancias concretas de espacio y tiempo. Todas las medidas y observaciones son inseparables de los instrumentos que se utilizan, están siempre vinculados a una porción de materia concreta dotada de ciertas propiedades concretas que, sin embargo, deben tomarse en abstracto. Es decir, han de ser generalizables para que sean válidas y aplicables a una multiplicidad de individuos de la misma clase.

Así, por ejemplo, la observación científica del movimiento de una pieza de hierro atraída por una barra magnética se hace siempre desde una perspectiva teórica o desde una hipótesis determinada que se ha adoptado previamente. En este caso, el movimiento de la pieza de hierro ya no se considera supeditado a las circunstancias singulares que acompañan a ese fenómeno concreto. Por esto, el efecto de atracción magnética no queda asociado a las propiedades individuales de una porción del mineral magnético dotado de determinada forma, color y tamaño, sino que por el contrario, a las sensaciones se superponen principios abstractos e hipótesis que operan en la mente del observador y le permiten superar los aspectos particulares de los hechos, es decir, aquellas circunstancias específicas que los circunscriben a la porción de materia concreta que tiene frente a sí. Por ello, en el ejemplo que comentamos, las propiedades magnéticas se toman en abstracto y se refieren intencionalmente a cualquier otro cuerpo de esas mismas características; no se atribuyen exclusivamente a los cuerpos materiales cuya presencia examinamos, aquí y ahora. En consecuencia, la obser- 
vación científica comporta una abstracción ${ }^{6}$, en virtud de la cual, se toman los aspectos o propiedades que son comunes a múltiples individuos y se prescinde de sus circunstancias accidentales, incluso, se eliminan aquellas otras propiedades que no atañen a la investigación en curso. Esta operación de abstracción, en el sentido que estamos considerando, es un primer paso para construir un esquema mental o modelo idealizado, cuyos rasgos característicos harán posible la aplicación del análisis matemático.

Esa operación de abstracción realizada con una finalidad científica, que recibe el nombre de idealización ${ }^{7}$, está en el comienzo de toda

6 Como ejemplo ilustrativo de lo que decimos, puede citarse el origen de la llamada electricidad galvánica, asociada a los experimentos que realizó Galvani, examinando los efectos dinámicos que se producían cuando dos piezas metálicas de metales de diferente clase se ponían en contacto con el nervio crural de una rana. $\mathrm{Al}$ principio, las observaciones de tal efecto hacían pensar, ante todo, en una causa fisiológica. Por lo cual, inicialmente, el fenómeno no podía catalogarse dentro de la clase de fenómenos producidos por electricidad estática, tales como los asociados a una barra de vidrio cargada de electricidad por frotamiento. Se comprende, así, que la atribución de un origen común a dos hechos en apariencia tan dispares requería la eliminación por abstracción de determinadas impresiones sensoriales inmediatas, ajenas a la electricidad.

7 Se han referido a ella, entre otros, algunos autores como R. Harré ("Idealization in Scientific Practice", Poznan' Studies in the Philosophy of the Sciences and the Humanities. Amsterdam, 1989, vol. 16: 183-191; C. Dilworth ("Idealization and the AbstractiveTheoretical Model of Scientific Explanation", Poznan' Studies in the Philosophy of the Sciences and the Humanities, Amsterdam, 1989, vol. 16: 167-181); L. Nowak ("Abstracts are not our constructs. The mental constructs are abstracts", Poznan' 
investigación científica. Pues los hechos individuales que se examinan, tal y como se perciben por los sentidos (los "hechos brutos", sin elaborar), no pueden ser objeto inmediato del estudio científico, sino que, tales hechos han de ser transformados mentalmente o idealizados hasta convertirse en objetos científicos ${ }^{8}$. Éstos guardan cierta semejanza con aquellos; al menos, los que están ligados a la materia y que percibimos por los sentidos. Por tanto, podemos afirmar que, aunque sean objetos formados por el pensamiento, hunden sus raíces en el mundo de los fenómenos naturales. Aquí, es apropiado señalar, además, que la operación de idealización tiene puntos en común con la abstracción geométrica, es decir, aquella operación, en virtud de la cual, se extraen mentalmente determinadas propiedades relativas a la forma de los cuerpos materiales y se prescinde del resto. La construcción idealizada de los objetos materiales que estudia la física sigue así un camino paralelo a la empleada en los objetos matemáticos y es razonable

Studies in the Philosophy of the Sciences and the Huminaties, Amsterdam, 1989: vol. 16: 193-206); y E. Mac Mullin ("Galilean idealization", Studies in History and Philosophy of Science. 1985, vol. 16, No. 3: 247-273). Con la noción de idealización suele expresarse el proceso seguido por la mente científica al construir una representación mental a partir de observaciones realizadas sobre determinado fenómeno.

8 Para Poincaré el "hecho científico" puede considerarse una traducción del "hecho bruto". "Todo lo que crea el científico en un hecho es el lenguaje en que lo enuncia" (Poincaré, H., La valeur de la science: 233. Citado por Antiseri, D., La Viena de Popper, Madrid, Unión Editorial, 2001: 119). 
considerarla como el comienzo del proceso de matematización -mediante la geometría - de los fenómenos físicos.

Según lo que acabamos de exponer, si se admite la construcción de objetos idealizados a partir de la captación sensible, no parece justificado hablar de "variables ocultas", de "cualidades ocultas", de "atributos latentes" o de nociones similares que algunos autores han utilizado para introducir términos científicos con la finalidad de salvar las apariencias, tal como denuncia Berkeley a propósito de la gravedad de los cuerpos pesados:

\footnotetext{
Cuando sostenemos cuerpos pesados, tenemos en nosotros mismos la sensación de esfuerzo, fatiga e incomodidad. Percibimos también en los cuerpos pesados que caen un movimiento acelerado hacia el centro de la Tierra: por medio de los sentidos [no percibimos] nada más. Mediante la razón, sin embargo, inferimos que hay una causa o principio de todos estos fenómenos, que vulgarmente se denomina gravedad. Pero puesto que la causa de la caída de los cuerpos pesados es invisible y desconocida, la gravedad en ese sentido no puede propiamente considerarse una cualidad sensible; es por tanto una cualidad oculta. Ahora bien, difícilmente, y ni siquiera difícilmente, puede concebirse qué sea una cualidad oculta o el modo como una cualidad oculta puede actuar o realizar algo. Así, mejor sería si, desechando la cualidad oculta, los hombres atendieran sólo a los efectos sensibles y, si prescindiendo en la reflexión de términos abstractos (por más que sean útiles en la discusión), la mente se dedicara a las cosas particulares y concretas, esto es, a las cosas mismas ${ }^{9}$ [Cursiva añadida].
}

9 Berkeley, G., De Motu, Madrid, Excerpta Philosophica. Facultad de Filosofía de la Universidad Complutense, 1993: 11. 
$\mathrm{La}$ argumentación es aplicable a cualquier otro ejemplo de la física y aún es más oportuna si se refiere a los fenómenos no mecánicos, como los electromagnéticos. En efecto, es preciso distinguir entre la percepción sensible y lo que mediante la razón auxiliada por el sentido se infiere en la observación. Mediante la razón que actúa en conjunción con los datos de los sentidos, es posible construir o definir determinados objetos o entidades que son útiles para la descripción del fenómeno en cuestión. En el ejemplo citado, el término gravedad (tomado del lenguaje común) sirve para referirse a la acción de la Tierra sobre los cuerpos, sin pretensión de dar una explicación científica. Precisamente, para dar esa explicación, la mecánica emplea la noción técnica de fuerza, pero no lo hace porque su existencia sea apreciable por los sentidos. El término científico como tal no responde a ninguna sensación, como la que produce el esfuerzo muscular o una impresión acústica, por ejemplo. La fuerza en cuanto término científico hace su aparición en la ciencia gracias a una definición apropiada que se atribuye a los cuerpos que intervienen, así la atracción gravitatoria es una acción mutua entre el cuerpo material y la Tierra. En este mismo sentido escribe Berkeley: "La fuerza es asimismo atribuida a los cuerpos; pero este vocablo está usado como si significara una cualidad conocida y distinta tanto del movimiento, figura y cualquier otra cosa sensible, como de toda afección del ser vivo" ${ }^{10}$. 
Con ello, desde una posición que hemos calificado de instrumentalista, Berkeley defiende la utilidad de los términos científicos únicamente cuando se aplican a "las cosas particulares y concretas", esto es, a las cosas materiales singulares, pero — según él— debido a su naturaleza abstracta no sirven para conocer "la naturaleza de las cosas, que son siempre singulares y concretas". Además, rechaza las nociones científicas que "ni son objeto de los sentidos, ni pueden ser concebidas por fuerza alguna del intelecto, ni representadas por la imaginación” y en consecuencia, según Berkeley, la ciencia debe eliminar el "Velo de las Palabras" para cumplir con su papel de dar a conocer el mundo físico ${ }^{11}$.

Sin embargo, sobre esta opinión, pensamos que los conceptos que formamos en nuestra mente a partir de la observación, pese a su idealidad, no sólo son útiles, en el sentido restrictivo que señala Berkeley, sino que además son construcciones mentales que pasan a formar parte de la teoría y son susceptibles de formalización matemática, pese a que se refieran a objetos no perceptibles. Esto ocurre con los conceptos mecánicos, tales como masa, fuerza, espacio, etc., o los empleados en electromagnetismo, tales como, campo, o carga eléctrica. Tendremos ocasión más adelante de referirnos a la construcción de términos científicos al analizar el método empleado por Maxwell. 145 . 
Esta misma hipótesis que comentamos encuentra justificación en la siguiente afirmación de Einstein:

Aún cuando [los conceptos científicos] son creaciones mentales libres, estas nociones y relaciones nos parecen más sólidas y más inalterables que la experiencia sensorial individual en sí misma, a la que jamás se le puede garantizar por completo, que no sea una ilusión o fruto de una alucinación ${ }^{12}$ [Cursiva añadida].

La construcción de los conceptos responde a la libre creación de su autor, pero no es arbitraria, sino que está restringida por los resultados experimentales a los que debe someterse. $\mathrm{Y}$, aunque su punto de partida radica en la experiencia sensible no consiste en meras impresiones sensoriales, por el contrario, es una elaboración mental ordenada que adquiere la categoría de un objeto de pensamiento, que llamamos objeto idealizado. Así ocurre, por ejemplo, con el concepto dinámico de fuerza, el cual supera las sensaciones que van asociadas al esfuerzo muscular que se realiza al empujar o arrastrar un objeto material. La noción científica de fuerza tiene un significado preciso y objetivo, exigido por el lenguaje científico, mientras que el ámbito propio de las sensaciones es subjetivo.

\section{EL MODELO HIDRODINÁMICO DE MAXWELL}

Este planteamiento epistemológico tiene especial interés cuando se refiere a los fenómenos electromagnéticos, cuya observación y estudio 
no forman parte de la experiencia ordinaria, al menos, con el mismo grado de apariencia sensible que los fenómenos mecánicos. Así, por ejemplo, la caída de un grave o el desplazamiento de un proyectil resultan más cercanos a la experiencia ordinaria que la atracción de una pieza de hierro por un imán. De ahí que las descripciones de estas últimas observaciones sean más abstractas que las de aquellos, o bien, tales descripciones están más alejadas de las imágenes visuales y de las impresiones sensoriales.

No obstante, a pesar de su limitación conceptual, las teorías físicas anteriores a Maxwell seguían basándose en los métodos que habían sido útiles en mecánica. Por ejemplo, la teoría newtoniana de acción a distancia servía para describir la atracción o repulsión entre cargas eléctricas. El nuevo modelo de campo electromagnético que hace su aparición en la física gracias al método analógico adoptado por Maxwell sustituirá con ventaja a las antiguas teorías. Desde el punto de vista epistemológico su análisis abre un camino apropiado para comprender mejor algunos aspectos del método científico, tales como la construcción del lenguaje científico y el estatuto cognoscitivo de las teorías.

Nuestro análisis se centrará sobre la investigación recogida en tres conocidos artículos científicos que fueron publicados por Maxwell entre 1856 y 1864, cuyos títulos y fechas de edición son los que siguen:

On Faraday's lines of force (1856)

On Physical lines of force (1862)

A Dynamical theory of electromagnetic field (1864) 
A lo largo de los nueve años que transcurrieron desde la primera publicación en 1856 hasta la tercera, en diciembre de 1864, su autor fue construyendo un modelo físico-matemático. En ese marco teórico encuentran su lugar apropiado los fenómenos eléctricos y magnéticos que Faraday había descrito en Researches in Electricity. Su elaboración es cada vez más compleja hasta llegar a una síntesis final que describe todos esos fenómenos mediante el análisis diferencial.

El modelo del campo electromagnético debía consistir en un entramado conceptual lógico que pudiera albergar en el plano teórico todos y cada uno de los sucesos eléctricos y magnéticos estudiados experimentalmente ya mencionados. Para acometer esa investigación, Maxwell comienza preguntándose cuál será el método más adecuado para obtener su objetivo. Se enfrenta con dos posibilidades, por un lado, podía adoptar una teoría ya establecida, como la que se refería a la existencia de fluidos eléctricos ${ }^{13}$, o por otro lado, podía seguir un método nuevo, independiente de otras teorías, que proporcione una base conceptual firme dentro de la ciencia física. En el primer caso, la teoría inicialmente adoptada impondría sus hipótesis y principios al

13 La electrización por frotamiento se asociaba a la emanación de supuestos fluidos eléctricos encerrados en el interior de los cuerpos (véase Dissertation concerning Electricity, 1742 by J. T. Desaguliers. Citado por Whittaker, E., A History of the Theories of Aether and Electricity, American Institute of Physics, 1951, vol. 7: 42). 
desarrollo posterior ${ }^{14}$. En el segundo caso, que fue el elegido por Maxwell, el proceso de construcción no resultaba comprometido con ningún planteamiento anterior.

Debemos, por consiguiente, descubrir un método de investigación que, en cada paso, permita a la mente sustentarse con firmeza sobre una clara concepción física, sin comprometerse con ninguna teoría basada en la ciencia física que tome de ella esa concepción en préstamo, de forma que, ni sea alejada del objetivo que persigue por sutilidades analíticas, ni trasportada más allá de la verdad por hipótesis favori$\operatorname{tas}^{15}$.

El método elegido tiene su fundamento en la analogía científica, en virtud de la cual, las teorías mecánicas van a adquirir un contenido electromagnético. En palabras de su autor, este tipo de analogía debe entenderse como "la similitud parcial entre las leyes físicas de una ciencia y las de otra que pueda utilizarse como ilustración recíproca entre ellas"16. El procedimiento comporta realizar una trasferencia formal entre ciencias diferentes. Su fundamento último reside en que todas las ciencias empíricas comparten, en mayor o menor medida, un

14 Acerca de las hipótesis de partida y del la adopción de modelos, véase Achinstein, P., "Scientific Discovery and Maxwell's Kinetic Theory", Philosophy of Science, 1987, vol. 54: 409-434.

15 Maxwell, J. C., The Scientific Papers of James Clerk Maxwell, Dover Publications, 2003, vol. 1: 156 .

16 Maxwell, J. C., The Scientific Papers of James Clerk Maxwell, Dover Publications, 2003, vol. 1: 156 . 
mismo lenguaje matemático. La descripción, mediante ese lenguaje, de los fenómenos naturales tiene la virtualidad de convertir las magnitudes que intervienen en cantidades numéricas, siendo tales valores numéricos el resultado de las operaciones experimentales necesarias para medir dichas magnitudes. Así ocurre, por ejemplo, con la similitud que existe entre la propagación de la luz en el espacio y las vibraciones mecánicas que se propagan en un medio elástico. En este caso, hay una semejanza de forma entre las vibraciones luminosas y las mecánicas. Tales analogías, sin embargo, no son aplicables con carácter general, es decir, no son válidas más allá de ciertos límites, dependiendo de la naturaleza de los fenómenos en cuestión.

La aportación metodológica inédita de Maxwell consiste en dar forma matemática a los resultados empíricos publicados por Faraday en Exprimental Researches in Electricity. En esa operación, los valores experimentales no pierden sus significados físicos. Es decir, se establecen conexiones lógicas valiéndose del cálculo matemático, sin abandonar las imágenes sensibles procedentes de las teorías mecánicas.

Mediante el uso de analogías de este tipo, he tratado de traer ante la mente, de una forma conveniente y manejable, aquellas ideas matemáticas que son necesarias para el estudio de los fenómenos de la electricidad [...] Mediante el método que he adoptado, espero mostrar que es evidente que no intento establecer ninguna teoría física de una ciencia en la que apenas he hecho un solo experimento y que el objeto de mi proyecto es mostrar cómo, mediante la estricta aplicación de las ideas y 
métodos de Faraday, puede ser claramente presentadas ante la mente matemática, la conexión de muy diferentes tipos de fenómenos que él ha descubierto to [Cursiva añadida].

En el trabajo de elaboración de la teoría del campo electromagnético se excluye, por tanto, todo propósito de investigar la naturaleza y constitución material de aquellos cuerpos que tienen propiedades eléctricas o magnéticas, tampoco se investiga el origen de la conducción eléctrica, ni del resto de los fenómenos. Por esto, cabe señalar que, desde el mismo punto de partida Maxwell adopta una postura metodológica que podría calificarse de instrumentalista, según la distinción realizada anteriormente. Puesto que, desde el principio su exposición manifiesta un propósito de describir con lenguaje matemático los datos empíricos disponibles, sin adoptar tesis ya establecidas sobre la naturaleza o sustancia de los hechos. Tampoco tiene la pretensión de descubrir las características físicas de los cuerpos, ni se plantea cuál es el origen, ni dónde se asientan las acciones eléctricas y magnéticas que se observan en el laboratorio. Por el contrario, parte de datos y medidas que proceden de las observaciones junto con las interpretaciones ya establecidas; en su mayor parte de orden cualitativo.

No obstante, la posición filosófica de Maxwell no responde a un premeditado enfoque instrumentalista. Desde luego, no consta así en sus escritos, por lo que su planteamiento metodológico, tal como queda explicito en la introducción al primer artículo citado, debe más 
bien calificarse de pragmático. Es decir, Maxwell elige el método analógico porque es el más útil para conseguir su propósito de construir una teoría que establezca un orden lógico en todos los hechos experimentales conocidos. Para lo cual, no duda en prescindir de todo planteamiento sobre el fundamento ontológico de la realidad material en la que se asientan tales fenómenos. No entra a considerar las propiedades constitutivas de los materiales eléctricos o magnéticos, sino que únicamente tiene en cuenta los efectos observables conocidos y cuantificables.

\subsection{DE LAS LÍNEAS DE CORRIENTE A LAS LÍNEAS MAGNÉTICAS}

Al excluir toda pretensión de conocer la estructura interna de los hechos empíricos, el papel fundamental de la construcción teórica recae sobre las semejanzas formales. Por esto, Maxwell utiliza la imagen que proporciona un líquido que discurre por su cauce, con el fin de ilustrar el trasporte de carga eléctrica a lo largo de un cuerpo conductor. Esta similitud entre dos situaciones tan diferentes sirve de soporte sensible a la mente para definir conceptos capaces de explicar por analogía el mecanismo interno de la conducción eléctrica. La analogía científica utiliza imágenes sensibles que toma prestadas de otra teoría conocida para acomodarlas a los resultados experimentales. En este sentido, podría considerarse que el modelo del fluido ideal desempeña un papel similar al del esquema geométrico de la astronomía ptolemaica para "salvar las apariencias". En uno y otro caso, los datos 
obtenidos como fruto de la observación científica deben encajar dentro del modelo, cualquiera que sea la realidad que describen.

Las amplias posibilidades de este método permiten, incluso, establecer analogías en casos muy dispares. Tal ocurre con la conducción del calor y las acciones a distancia, sean gravitatorias o eléctricas. Esto, a pesar de que las leyes que rigen las fuerzas de atracción o de repulsión entre cargas eléctricas son muy diferentes de las que rigen la conducción del calor. En este último, las magnitudes que intervienen (según los términos utilizados por Maxwell) son la fuente de calor, la temperatura y el flujo de calor.

Sin embargo encontramos que las leyes matemáticas del movimiento uniforme del calor en un medio homogéneo son idénticas en forma a las de atracción, que varían inversamente al cuadrado de la distancia. Sólo tenemos que sustituir la fuente de calor por el centro de atracción, el flujo de calor por la aceleración en cualquier punto debida a la fuerza y la temperatura, por el potencial, y así la solución de un problema sobre atracción se transforma en un problema sobre calor ${ }^{18}$.

Por tanto, el método analógico pone de relieve que en el plano del formalismo matemático es indiferente qué tipo de entidad origina el hecho físico que se estudia. Por esto, los términos científicos que caracterizan los fenómenos que son homólogos en la analogía guardan entre sí un paralelismo formal. No se trata de una similitud que afecte sólo al conjunto, pues se establece entre las magnitudes correspon- 
dientes que aparecen en las leyes que rigen cada uno de los fenómenos análogos. Y esto, aunque el significado de esos términos esté restringido a su propio ámbito. Así, en el ejemplo citado sobre la teoría del calor, el papel que juega la temperatura en la conducción del calor es análogo al que juega el potencial eléctrico en el campo eléctrico; sin embargo, el significado propio de cada uno es muy diferente, pues viene asignado por el tipo de fenómeno en el que se ha definido.

Este enfoque metodológico que hemos llamado pragmático guiará el trabajo de Maxwell en la construcción de la teoría del campo electromagnético. Este pragmatismo pervive a lo largo de las tres etapas del desarrollo del modelo hidrodinámico que se describen en cada uno de los tres artículos referidos. En el primero de ellos, Maxwell indica el proceso que va a seguir:

Primero, describir un método por el cual el movimiento de tal fluido pueda ser claramente concebido; segundo, desarrollar las consecuencias derivadas de suponer el movimiento sujeto a ciertas condiciones y señalar la aplicación del método a algunos de los fenómenos menos complicados de la electricidad, del magnetismo y del galvanismo; y, finalmente, mostrar cómo pueden ser claramente concebidas las leyes de atracción y de acción inductiva de barras magnéticas y de corrientes, mediante extrapolación de estos métodos y de la introducción de otra idea debida a Faraday, sin hacer ninguna suposición sobre la naturaleza física de la electricidad, o sin añadir nada que no haya sido ya probado por la experimentación ${ }^{19}$ [Cursiva añadida].

19 Maxwell, J. C., The Scientific Papers of James Clerk Maxwell, Dover Publications, 2003, vol. 1: 159. 
En este programa de investigación queda excluida la aplicación directa del análisis matemático a los resultados experimentales, ya que supondría prescindir del significado físico y, con ello, perder la vinculación con los hechos experimentales. Por el contrario, el esquema hidrodinámico elegido está dotado de un significado físico conocido que podrá ser interpretado convenientemente para describir las interacciones electromagnéticas, cuyos mecanismos internos no son observables.

Hemos de subrayar que tal fluido es una construcción imaginada, un fluido ideal, supuesto como un medio continuo formado por partículas en movimiento. Según la teoría hidrodinámica, tales partículas describen trayectorias lineales, llamadas "líneas de corriente", las cuales guardan un paralelismo evidente con las "líneas de fuerza"20 definidas por Faraday. El fundamento de tal analogía entre las "líneas de corriente" y las "líneas de fuerza" reside en la naturaleza geométrica que ambas entidades físicas comparten. En lo sucesivo, dichas representaciones de índole geométrica se convertirán en el eje conductor de todo el desarrollo conceptual posterior.

20 Los pequeños fragmentos de hierro inicialmente dispersos aleatoriamente sobre la cara superior de una hoja de papel en posición horizontal se orientan formando líneas geométricas, cuando se acerca por la cara inferior del papel una barra magnética (Figura 1). Faraday utiliza indistintamente los nombres: line of magnetic force, magnetic lines of force, o magnetic curve (Faraday, M, Experimental Research in Electricity, New York, Dover, 1965, vol. 3: 2). 
Es oportuno señalar ahora que el paralelismo entre un concepto cinemático (la trayectoria de una partícula material en movimiento y una línea de fuerza magnética) radica en la forma geométrica que ambas tienen en común, pero, a su vez, tal similitud presupone la idealización de ambas entidades físicas. Puesto que, ateniéndonos únicamente a la observación visual, ni las "líneas de corriente", ni las "líneas de fuerza" son apreciables por los sentidos. En el caso del movimiento del fluido, lo que se ve es una masa líquida que se desplaza en una dirección, no se ven las partículas, ni mucho menos se perciben las trayectorias individuales que siguen. En realidad, el mismo concepto de partícula es una construcción idealizada que se hace aislando mentalmente una pequeña porción de líquido. Aún menos, pueden hacerse visibles las "líneas de fuerza", puesto que son también una construcción mental idealizada sugerida por la disposición geométrica que toman las limaduras de hierro sometidas a la acción de una barra magnética como muestra la Figura 1.

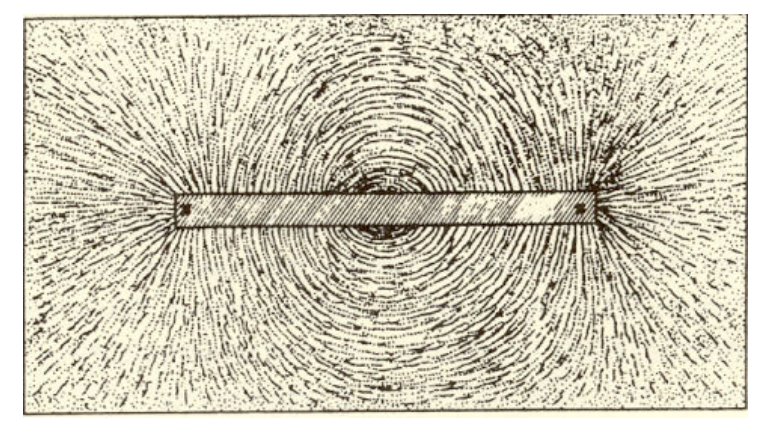

Figura 1. Formación de curvas magnéticas. 


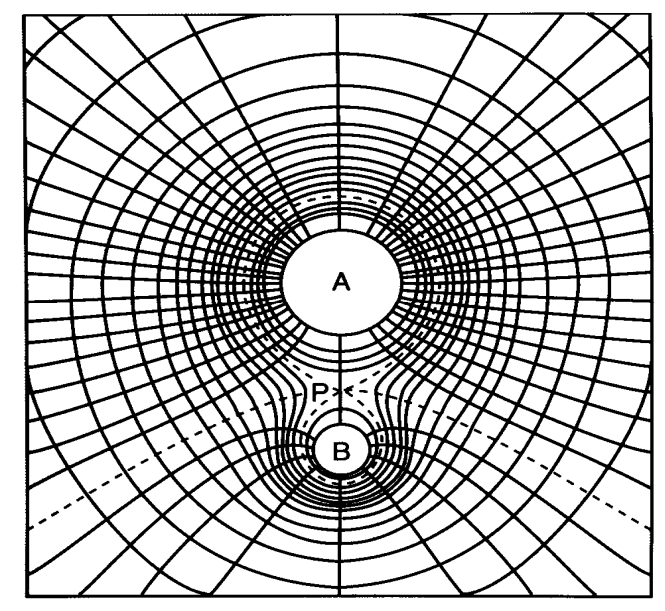

Figura 2. Diagrama de líneas de fuerza y superficies equipotenciales.

La analogía formal establecida por Maxwell concibe el medio donde se produce la interacción electromagnética como un fluido ideal en el que, sin embargo, de hecho, no hay ningún trasporte de materia. En él, se suponen las "líneas magnéticas" llenando todo el volumen del fluido ideal, cuyas direcciones en cada punto indican la dirección de la fuerza magnética en ese mismo punto ${ }^{21}$. Posteriormente, las líneas de fuerza serán transformadas en tubos de fuerza, un nuevo artificio simbólico que representa la dirección y la intensidad de la fuerza magnética. Con ello se establece una nueva analogía con la velocidad del fluido que circula por un tubo de sección variable y la circulación

21 Maxwell, J. C., The Scientific Papers of James Clerk Maxwell, Dover Publications, 2003, vol. 1: 158 . 
de un fluido hipotético, el "fluido magnético", determinado en cada punto por su dirección e intensidad. A partir de ese esquema ideal, la construcción del modelo prosigue imaginando ahora un conjunto superficies perpendiculares a las superficies cilíndricas anteriores, cuya función consiste en representar las zonas del fluido de igual presión (La Figura 2 ilustra este esquema en dos dimensiones).

Mediante este tipo de representaciones visuales, Maxwell construye un entramado geométrico formado por superficies ortogonales que llenan todo el medio fluido. A partir de ahí, está en condiciones de abordar la transposición de las magnitudes mecánicas (que definen el movimiento del fluido) en las nuevas magnitudes que describirán los fenómenos electromagnéticos.

\footnotetext{
Ahora tengo que mostrar cómo la idea de líneas de movimiento de fluido según se han descrito más arriba puede ser modificada de forma que sea aplicable a la ciencia de la electricidad estática, al magnetismo permanente, a la inducción magnética y a las corrientes galvánicas uniformes, dejando las leyes del electromagnetismo para una consideración especial ${ }^{22}$.
}

Así, por ejemplo, siguiendo la analogía hidrodinámica, la presión que se origina en el interior del medio fluido tiene su homóloga en el potencial eléctrico. Una vez más, Maxwell hace caso omiso del significado físico original de las magnitudes, manteniéndose fiel a su propósito de construir un modelo ideal sin entrar en hipótesis sobre el 
fundamento físico de los fenómenos eléctricos. No se plantea siquiera cuál es la naturaleza de la carga eléctrica, ni se pregunta el porqué de las relaciones de proporcionalidad entre carga, fuerza y distancia; por el contrario, las acepta como algo probado experimentalmente. $\mathrm{Y}$ toma la mera estructura formal, es decir, utiliza los símbolos de la ecuación que define el movimiento del fluido para establecer el paralelismo entre las magnitudes eléctricas y las hidrodinámicas. Por esto, una vez realizada la trasposición de significados, la presión en cada punto del medio fluido, vendrá a significar el potencial electricidad. Igualmente, la magnitud que representa la fuente ${ }^{23}$ (los puntos donde se origina el fluido) tiene su homóloga en la carga eléctrica.

En esta transferencia de significados, las relaciones matemáticas que se refieren a una y a otra ciencia son iguales. Es decir, en hidrodinámica la presión $p$ en un punto del medio fluido es proporcional al valor de la fuente $S$ e inversamente proporcional a la distancia $r$ (simbólicamente, $p=S / r$ ). En virtud de la analogía es $V=Q / r$, siendo el potencial eléctrico $V$ en un punto situado a distancia $r$ de una carga Q. La distancia es una magnitud de naturaleza geométrica, que comparten ambos fenómenos, en uno de ellos, indica la distancia a la fuente, y en el otro, la distancia a la carga.

Importa subrayar con carácter general que en las ecuaciones algebraicas anteriores todos los términos que aparecen quedan equiparados

23 El término fuente debe entenderse como una magnitud que indica el caudal de fluido originado. 
a magnitudes matemáticas. Es decir, por su naturaleza simbólica, los términos citados que se refieren a conceptos de naturaleza física (la carga y el potencial, en el medio eléctrico y la fuente y la presión, en el medio fluido) juegan el mismo papel que el tercer término, la distancia, que tiene naturaleza geométrica. Esto justifica la decisión pragmática de Maxwell de prescindir de los significados físicos de los símbolos, tomando las expresiones matemáticas, como estrictamente simbólicas. En este mismo sentido Thomas K. Simpson afirma que las magnitudes simbólicas de las ecuaciones pertenecen a dos dominios de distinta naturaleza, esto es, el eléctrico y el mecánico. En consecuencia, "lo que obtenemos es una relación puramente cuantitativa $[\ldots]$ que se maneja (pues, nuestro modelo es sólo mental) para ajustar nuestros pensamientos"24.

Por lo cual, es consecuente afirmar que el método adoptado por Maxwell parece avalar la concepción instrumentalista de la ciencia, puesto que prescinde totalmente de hipótesis sobre la constitución de los objetos materiales o sobre su existencia real y se centra únicamente en el aspecto operacional, construyendo un modelo ideal basado en las leyes que rigen en mecánica de fluidos.

24 Simpson, T. K., Maxwell on the Electromagnetic Field, London, Rutgers University Press, 1998: 138. 
Naturaleza y Libertad. Revista de estudios interdisciplinares. Número 1, 2012. ISSN: 2254-9668

\subsection{COMPROBACIÓN DEL MODELO}

Con el fin de precisar la extensión y utilidad del método analógico vamos a referirnos a alguna de las consecuencias del modelo que Maxwell analiza para demostrar la validez de su construcción teórica. En los últimos apartados de On Physical lines of force se recogen las aplicaciones del modelo ideal a una serie de observaciones experimentales. Éstas ponen de manifiesto, tanto la coherencia lógica de la teoría, como su comprobación empírica. Utilizando la analogía hidrodinámica, el modelo sirve para explicar las propiedades de los materiales dieléctricos y las de los cuerpos magnéticos permanentes. Asimismo, da cuenta de los fenómenos de inducción paramagnética, diamagnética y magnetocristalina. También, es capaz de explicar en términos mecánicos la conducción eléctrica, la fuerza electromotriz, incluso, la fuerza a distancia producida por circuitos de corriente y las corrientes eléctricas originadas por inducción magnética.

El modelo hidrodinámico explica la inducción eléctrica en ciertos materiales. Este fenómeno había sido estudiado por Faraday y se produce cuando un cuerpo eléctricamente cargado (por ejemplo, una barra de vidrio dotada previamente con cierta cantidad de electricidad estática) es capaz de electrizar a otro cuerpo inicialmente descargado (por ejemplo, una porción de resina de ámbar). El efecto de inducción electrostático depende de la capacidad inductiva del medio donde se 
hallan inmersos ambos cuerpos ${ }^{25}$. En conformidad con el modelo, la capacidad inductiva es análoga a la resistencia que experimentan en su desplazamiento las partículas que se mueven en el interior del fluido.

Si suponemos que en nuestra analogía del fluido en un medio resistente, la resistencia es diferente en medios diferentes, entonces haciendo la resistencia menor obtenemos la analogía a un dieléctrico que conduzca más fácilmente las líneas de Faraday $^{26}$ [Cursiva añadida].

La analogía se extiende, no sólo a la trasmisión de acciones eléctricas o magnéticas, sino también a su origen. En efecto, Maxwell establece similitudes entre el polo norte y el polo sur de un imán permanente y las fuentes y sumideros de un fluido. De forma que, según el modelo, las líneas magnéticas que salen del polo norte y terminan en el polo sur son análogas a las líneas de corriente de un fluido, que se originan en las fuentes y terminan en los sumideros.

En resumen, de acuerdo con la primera versión del modelo del fluido ideal es posible, mediante conceptos mecánicos, explicar todas las propiedades eléctricas y magnéticas estudiadas por Faraday, con excepción de la inducción electromagnética. Fenómeno que se manifiesta por la aparición de una corriente en un cuerpo conductor, cuando se

25 Faraday se refiere a esa propiedad en "Naturaleza de la inducción eléctrica" publicado en Researches (serie XI).

26 Maxwell, J. C., The Scientific Papers of James Clerk Maxwell, Dover Publications, 2003, vol. 1: 177 . 
desplaza en la proximidad de una barra magnética (siempre que ese desplazamiento sea en dirección transversal a las líneas magnéticas producidas por la barra). Según la explicación cualitativa dada por $\mathrm{Fa}^{-}$ raday, el efecto se produce en virtud de una alteración del espacio libre donde se encuentran el conductor y la barra magnética. Esa alteración da lugar a un nuevo estado del medio que llamó electro-tónico ${ }^{27}$, por analogía con el estado de tensiones que se producen en el interior de un sólido elástico sometido a fuerzas exteriores que alteran su estructura y producen pequeños esfuerzos internos repartidos por todo el material.

La idea de estado electro-tónico, sin embargo, no se me presentaba en sí misma en tal forma que su naturaleza y propiedades puedan ser explicadas sin referencia a meros símbolos y por tanto, en la próxima investigación me propongo utilizarlos libremente [...]. Mediante un estudio preciso de las leyes de los sólidos elásticos y de los movimientos de fluidos viscosos. Confio descubrir un método para formar una concepción mecánica de este estado electro-tónico adaptado al razonamiento general ${ }^{28}$.

Una vez más, el trabajo de Maxwell consistirá en dar forma matemática a esa concepción cualitativa, utilizando una analogía basada en las propiedades elásticas de los materiales. Para lo cual, modificará la primera versión del modelo del fluido. Esta segunda versión, publi-

27 Cat, J., "On Understanding: Maxwell on the Methods of Illustration and Scientific Metaphor", Studies in History and Philosophy of Modern Physics 2001, vol. 32, No. 3: 395-441.

28 Maxwell, J. C., The Scientific Papers of James Clerk Maxwell, Dover Publications, 2003, vol. 1: 187-188. 
cada con el título On Physical lines of force, sugiere que Maxwell va a investigar la posible naturaleza física de esas "líneas de fuerza", aunque, en el artículo anterior ha considerado las "líneas de fuerza" como idealizaciones o representaciones geométricas. Por lo cual, este nuevo enfoque de la investigación hace pensar que Maxwell no descarta la posibilidad de que tales "líneas de fuerza" tengan algún tipo de consistencia real. Entonces, no serían representaciones simbólicas ideales, sino manifestaciones naturales de los fenómenos electromagnéticos. A este propósito, señala Simpson ${ }^{29}$, cabe preguntarse si Maxwell está buscando algo o, por el contrario, se encuentra construyendo un mundo que se acomode a su profunda convicción. A nuestro parecer, lejos de todo planteamiento filosófico, ante todo predomina la actitud pragmática decidida a construir una teoría que dé unidad a la gran variedad de fenómenos experimentales conocidos, sin excluir una posible base real que inspire el esquema geométrico establecido. De hecho, en el transcurso de la investigación antepone la construcción de la teoría a la investigación sobre la naturaleza física de los fenómenos naturales. Aunque, el título del artículo sugiere ese propósito, referido a la naturaleza física de las líneas de fuerza, de hecho, a lo largo del desarrollo se impondrá de nuevo la determinación de construir un formalismo teórico, salvando la realidad física de los fenómenos electromagnéticos. 
Al comienzo de On Physical lines of force, Maxwell se refiere a la "hermosa ilustración" que ofrecen los filamentos de hierro en el seno del campo magnético. Esta imagen "de un modo natural nos hace pensar en las líneas de fuerza como algo real" [...] naturally tends to make us think of the lines of force as something real [...]. Es decir, como algo que se asienta en el medio de tal modo que, allí donde se manifiestan las líneas de fuerza ha de existir un estado físico de tal naturaleza que sea capaz de actuar sobre minúsculas piezas de hierro, orientándose en la forma que se aprecia a simple vista. Esta característica configuración de la materia lleva a Maxwell a plantearse la investigación en los términos siguientes:

Mi objetivo en este artículo es aclarar el camino para la especulación en esta dirección [la de la naturaleza física del medio], investigando los resultados mecánicos de ciertos estados de tensión y movimiento en un medio y comparar éstos con los fenómenos observados del magnetismo y de la electricidad ${ }^{30}$ [Cursiva añadida].

Para alcanzar ese objetivo, Maxwell introduce una serie de modificaciones en el modelo del fluido ideal. Concibe, ahora, un medio fluido dotado de propiedades elásticas, en el que existen tensiones internas producidas por el propio movimiento de la masa fluida, tal y como ocurre en el movimiento de un fluido real. Para establecer la analogía apropiada ahora debe aplicar la teoría mecánica relativa a los

30 Maxwell, J. C., The Scientific Papers of James Clerk Maxwell, Dover Publications, 2003, vol. 1: 452 . 
medios elásticos, introduciendo la idea de vórtice molecular. Entiende por tal concepto, una porción cualquiera de masa fluida que gira alrededor de su propio eje de simetría obligado por las presiones que ejercen las regiones del fluido que están en contacto con dicho vórtice.

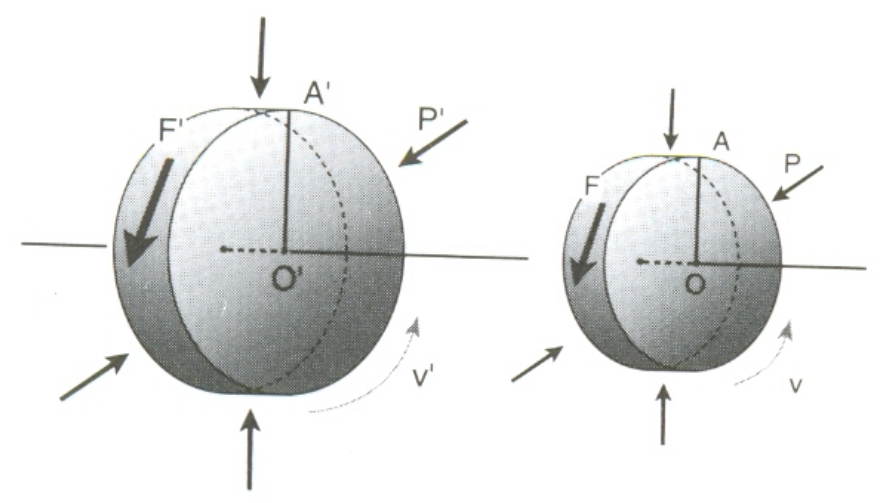

Figura 3. Representación de vórtices como elementos diferenciales.

Esta nueva entidad física responde a una nueva idealización realizada a partir de los "torbellinos" o "remolinos" que se forman, por ejemplo, en un caudal del agua que discurre por su cauce, siguiendo un régimen no laminar. Esta nueva representación de origen sensible se articula con las "líneas magnéticas", definidas en la primera etapa del modelo, de tal forma que, cada línea de fuerza coincide con el eje de cada vórtice. Por tanto, esas porciones de materia repartidas por todo el volumen del fluido en movimiento giran alrededor de las líneas de fuerza. Finalmente, como había sugerido Faraday, las líneas de fuerza tendrán un carácter bipolar, es decir, cada una de ellas está asociada 
con una "tensión" que se ejerce en el sentido longitudinal marcado por la propia línea (con el sentido de avance de las partículas del fluido) y una "presión" ejercida en sentido ecuatorial y producida por las acciones de la masa de fluido que rodea a cada vórtice.

En virtud de esta nueva idealización geométrica, ilustrada en la Figura 3, cada vórtice o porción de fluido en movimiento rotatorio (denominado por Maxwell "elemento del medio" ${ }^{31}$ ) puede ser ahora representada como un elemento de volumen y puede ser sometido al análisis diferencial. Con esta nueva herramienta matemática y las leyes físicas de la hidrodinámica, Maxwell obtiene una ecuación general que relaciona la "fuerza por unidad de volumen" con otras magnitudes del medio, tales como la "velocidad", la "densidad" y la "presión".

Se establece así un nexo entre los símbolos del cálculo diferencial y las magnitudes mecánicas que describen el movimiento del fluido. A continuación, Maxwell reagrupa los términos de las expresiones matemáticas según su conveniencia (siempre respetando la sintaxis propia del lenguaje matemático) hasta llegar a una ecuación que es capaz de proporcionar una clara interpretación física. Es decir, establece una correspondencia entre dos planos, por un lado, el de los signos matemáticos $\mathrm{y}$, por otro, el de las representaciones geométricas del modelo. A este respecto, Simpson subraya la habilidad de Maxwell para realizar transformaciones algebraicas, mediante las cuales encuentra un significado a partir de un simple conjunto de símbolos sin 
sentido $^{32}$, estableciendo así un paralelismo formal entre la estructura simbólica no figurativa del lenguaje diferencial y la representación gráfica del modelo. El resultado obtenido queda recogido en una expresión final en términos físico-matemáticos que indica cómo depende la presión ejercida sobre cada partícula del fluido en función de otras magnitudes, tales como la velocidad, la masa, etc.

En consecuencia, debido a la analogía, las ecuaciones y los términos mecánicos se trasfieren al dominio conceptual del electromagnetismo. Las magnitudes que tenían un significado mecánico reciben un nuevo sentido y algunos de los nuevos conceptos mantienen los nombres de procedencia (denotando con ello su origen mecánico), por ejemplo, la cantidad de materia magnética [amount of magnetic matter ${ }^{33}$ ] (como tal inexistente); o bien, la intensidad de fuerza magnética [intensity of the magnetic force], donde el término "fuerza" se toma prestado de la mecánica, atribuyéndole ahora un significado más amplio.

\subsection{SOBRE LA PRETENDIDA REALIDAD DE LAS LÍNEAS DE FUERZA}

Las explicaciones obtenidas hasta aquí tienen una validez supeditada al modelo ideal definido. Maxwell sabe que se trata de “especulaciones" (con el sentido que suele dar a este término), por lo

32 Simpson, T. K., Maxwell on the Electromagnetic Field, London, Rutgers University Press, 1998: 242.

33 Maxwell, J. C., The Scientific Papers of James Clerk Maxwell, Dover Publications, 2003, vol. 1: 459 . 
que intenta descubrir la naturaleza física de las líneas de fuerza, tal como sugiere el título del artículo, On Physical lines of force ${ }^{34}$. Así mismo, debe esclarecer qué principios físicos dan lugar a ese comportamiento del fluido. Se pregunta por las causas mecánicas que producen la rotación de los vórtices, teniendo en cuenta que su "velocidad circular" ha de ser proporcional a la intensidad de la fuerza magnética y que su "densidad" en el fluido ideal ha de ser proporcional a la inducción magnética del medio. Maxwell concede abiertamente que aún no ha dado respuesta a este tipo de preguntas que hacen referencia a su naturaleza física:

Aún no hemos dado respuestas a las cuestiones, ¿cómo se ponen en rotación los vórtices? y ¿por qué están distribuidos conforme a las leyes conocidas de las líneas de fuerza sobre barras magnéticas y corrientes? ${ }^{35}$.

Para responder a esas cuestiones, centrará su investigación en los vórtices moleculares que llenan todo el fluido y en concreto debe averiguar la conexión física entre tales vórtices y las corrientes eléctricas,

34 Maxwell "se proponía una teoría que debía considerarse como un candidato a 'teoría verdadera'. Podía ser falsa, es cierto, pero también, verdadera; esto es, describir la auténtica realidad física. El estatus ontológico de ambos artículos es, por consiguiente, muy diferente" (Sánchez Ron, J. M. (ed.), James Clerk Maxwell. Escritos Cientificos, Madrid, Consejo Superior de Investigaciones Científicas, 1998: XLVI.

35 Maxwell, J. C., The Scientific Papers of James Clerk Maxwell, Dover Publications, 2003, vol. 1: pp. 467-8. 
pues ahí supone que debe encerrarse la clave que explica el mecanismo de la inducción electromagnética.

De hecho, ahora, hemos de investigar acerca de la conexión de estos vórtices con las corrientes eléctricas, aunque aún tenemos duda sobre la naturaleza de la electricidad, si consiste en una sustancia, en dos sustancias, o no existe sustancia alguna, o bien en qué difiere de la materia y cómo está relacionada con ella ${ }^{36}$ [Cursiva añadida].

Maxwell no afronta directamente la investigación sobre la naturaleza física de las líneas de fuerza, sino que, primero trata de explicar cuál es papel que desempeñan los vórtices en la inducción electromagnética. $\mathrm{Y}$, de modo un tanto sorprendente, trata de hacerlo mediante la construcción mental de un nuevo modelo mecánico, al que se refiere William Berkson en los términos siguientes:

El modelo mecánico del campo electromagnético de Maxwell es uno de los más imaginativos pero menos verosímiles que nunca se hayan inventado. [...] Continuamente atribuye a su modelo propiedades descabelladas, sin que quepa en cabeza humana que haya un sistema real que posea tales propiedades ${ }^{37}$.

En realidad, se trata de un artificioso mecanismo que fue sugerido a Maxwell por William Thomson. Ambos científicos compartían el método analógico y pretendían explicar los fenómenos físicos me-

36 Maxwell, J. C., The Scientific Papers of James Clerk Maxwell, Dover Publications, 2003, vol. 1: 468.

37 Berkson; W., Las teorias de los campos de fuerza. Desde Faraday hasta Einstein, Madrid, Alianza Editorial, 1981: 188. 
diante modelos mecánicos. El mecanismo imaginado para hacer comprensible la inducción magnética estaba formado por un conjunto de piezas que se movían a modo de engranajes, como ilustra la Figura 4, que reproduce el dibujo original de Maxwell.

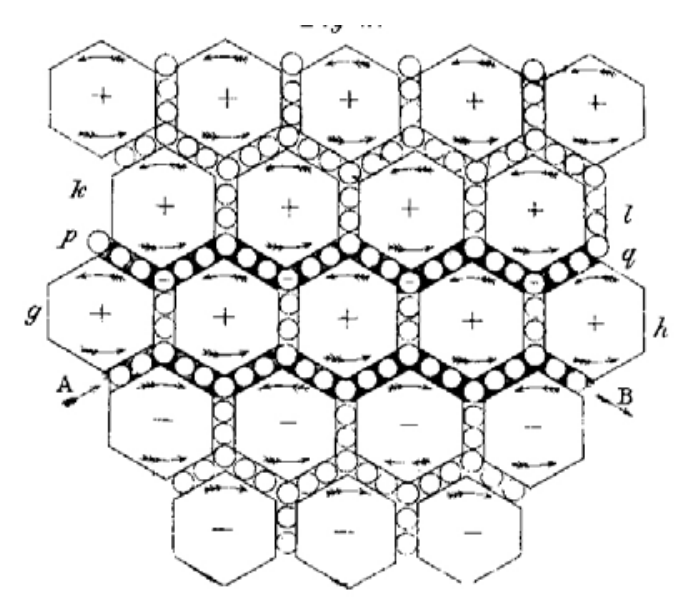

Figura 4. El modelo de vórtices.

¿Qué tipo de consistencia real atribuía su autor a este ingenioso modelo? A juzgar por su nota a pie de página al artículo que comentamos, investigó la posibilidad de que tal mecanismo formase parte de la estructura microscópica de la materia ${ }^{38}$. Así parece deducirse de sus

38 A propósito de esta investigación, A. F. Chalmers descarta una posición de realismo ingenuo por parte de Maxwell. Entendiendo por tal realismo el que admite que todo fenómeno observable es manifestación de las propiedades de un único 
comentarios sobre el pequeño tamaño de los vórtices comparado con el de una molécula de materia ordinaria ${ }^{39}$.

El momento angular del sistema de vórtices depende de su diámetro medio, de forma que si el diámetro fuera apreciable podríamos esperar que una barra magnética se comportase como si contuviese un cuerpo giratorio en su interior y así, podría ser detectada la existencia de esta rotación mediante experimentos sobre la libre rotación de una barra magnética. Yo he hecho experimentos para investigar esta cuestión, pero aún no he probado completamente el dispositivo ${ }^{40}$ [Cursiva añadida].

Maxwell parece tan identificado con su modelo que, a pesar de haber sido ideado sin base real y sin estar avalado por medidas experimentales, intenta detectar la existencia de los vórtices mediante la medida de su momento angular ${ }^{41}$. Es decir, por el mismo procedimiento que se utilizaría en el laboratorio con un cuerpo de dimensiones macroscópicas que gira alrededor de un eje fijo. Pero, el mecanismo que tendría las mismas propiedades y sería descrito en los mismos términos que los sistemas mecánicos, directamente observables. (Chalmers, A. F., "Maxwell's methodology and his applications of it to electromagnetism", Studies in History and Philosohy of Science 1973, vol. 4, no. 2: 155).

39 Maxwell, J. C., The Scientific Papers of James Clerk Maxwell, Dover Publications, 2003, vol. 1: 485.

40 Maxwell, J. C., The Scientific Papers of James Clerk Maxwell, Dover Publications, 2003, vol. 1: 485-6.

41 El momento angular, también llamado momento de la cantidad de movimiento, depende de la distribución de la masa del sólido respecto del eje de giro y de la velocidad de rotación del cuerpo. 
fracasado intento de revelar la existencia de los vórtices no le disuade de utilizar el esquema mecánico imaginado para explicar el fenómeno de inducción electromagnética.

Teniendo en cuenta el enfoque metodológico que hemos adoptado, importa analizar en qué consiste la analogía entre el modelo y el fenómeno de inducción. En primer lugar, recordemos que toda corriente eléctrica que circula por un conductor produce a su alrededor un campo magnético. Si la corriente es de intensidad constante, el campo magnético también permanece constante. Pero, si la intensidad de la corriente (llamada inductora) varía en función del tiempo, también lo hará el campo magnético asociado. Esta variación del campo magnético genera, a su vez, una corriente eléctrica inducida. Hay por tanto, una doble interacción entre electricidad y magnetismo, que se produce en un sentido y en el contrario. Por un lado, una variación de intensidad de corriente, genera un campo magnético variable y, en sentido opuesto, esa variación de campo magnético induce una corriente eléctrica. Pues bien, el mecanismo ideado por Maxwell pone de manifiesto de forma visual (mecánica) esa interrelación entre la corriente eléctrica y el campo magnético. Y lo hace, mediante los desplazamientos laterales de esferas mecánicas que representan las corrientes y el giro de piezas de forma hexagonal que representan los vórtices magnéticos (véase, Figura 4). No es de extrañar que un mecanismo tan poco refinado e ideado con la pretensión de explicar un fenómeno tan complejo provocase en Pierre Duhem (1861-1916) el irónico comentario siguiente: 
Creíamos entrar en la tranquila y ordenada estancia de la razón, sin embargo nos encontramos en una fábrica ${ }^{42}$.

La crítica anterior del científico francés no está sólo dirigida a un caso concreto, sino que, se refería a la escuela inglesa en general, cuyo método de investigación se basaba en la construcción de analogías mecánicas; por el contrario, la escuela francesa tenía preferencia por los razonamientos abstractos ${ }^{43}$. El modelo mecánico resultó de gran utilidad, pues como afirma Berkson: "la fábrica, sin embargo, dio algunos productos notables, a saber, las ecuaciones del campo electromagnético y la teoría electromagnética de la luz" ${ }^{4}$. El modelo de Maxwell no debe considerarse como una representación de la "realidad física" sino como un procedimiento ingenioso de expresar relaciones conceptuales entre fenómenos electromagnéticos utilizando un lenguaje mecánico, el cual, no obstante, sirvió para orientar la investigación posterior hasta la formulación final de la teoría.

En lo sucesivo, Maxwell no hará ninguna mención a la supuesta existencia física de los vórtices y seguirá desarrollando el método

42 Duhem, P., The Aim and Structure of Physical Theory, Princeton University Press, New Jersey, 1999: 71.

43 Sobre la crítica de Duhem al método científico utilizado por Maxwell, véase Ariew, R.; Barker, P., "Duhem on Maxwell: A Case-Study in the Interrelations of History of Science and Philosophy of Science", Proceedings of the Biennial Meeting of Science Association, 1986: 145-156.

44 Berkson; W., Las teorias de los campos de fuerza. Desde Faraday hasta Einstein, Madrid, Alianza Editorial, 1981: 191. 
analógico ya iniciado. En realidad, tal investigación le habría exigido un análisis microscópico de la materia, utilizando medios técnicos que entonces no existían.

Pero, en nuestro análisis el modelo mecánico tiene utilidad como ilustración de la interconexión entre corrientes y vórtices magnéticos. Si prescindimos de la materialidad de las piezas y de los engranajes del modelo y tomamos sólo la función del mecanismo como trasmisor de movimientos, resulta una conexión entre dos tipos de movimientos, uno de rotación y otro de traslación, que se produce en todos y cada unos de los puntos del espacio. El movimiento de rotación representa la intensidad del campo magnético y el de traslación, o desplazamiento, simboliza las corrientes inducida e inductora.

Esa conexión entre ambos movimientos, es posteriormente traducida al lenguaje matemático, llegando a obtener una función matemática (conocida actualmente con el nombre de "rotacional" ${ }^{45}$ ) que depende de la variación de la velocidad de los vórtices. Así pues, las entidades matemáticas no están significadas directamente en el fenómeno físico observable, sino en el modelo mecánico y en éste cobran sentido. En definitiva, la analogía mecánica y la ideación de un modelo apropiado han servido de puente entre los hechos empíricos y la construcción matemática. Aquellos, una vez idealizados, han que-

45 La relación aludida es una de las ecuaciones del campo electromagnético: $\operatorname{rot} B=\mu$ $J$ en notación actual. El primer miembro es la forma simbólica que se refiere a la rotación (rotacional de $B$ ), el segundo es el producto de la constante magnética del medio por la densidad de corriente $J$. 
Naturaleza y Libertad. Revista de estudios interdisciplinares. Número 1, 2012. ISSN: 2254-9668

dado inmersos en un modelo y posteriormente descritos como símbolos matemáticos, sin pérdida de significado físico, haciendo así posible las aplicaciones y la verificación experimental de la teoría.

\section{DEL FLUIDO IDEALIZADO AL CAMPO ELECTROMAGNÉTICO}

\subsection{EL TRIUNFO DEL FORMALISMO}

El tercer artículo A Dynamical theory of the electromagnetic field, publicado en The Royal Society Transactions, marca una clara diferencia respecto a los dos anteriores. Ahora, el modelo hidrodinámico se considera bajo otra perspectiva muy diferente. Sin prescindir totalmente de su significación mecánica, la investigación se centra en la noción de energía y se estudian las acciones mecánicas bajo la perspectiva operacional de la Mecánica Analítica, la cual supera la descripción geométrica de Newton.

Maxwell comienza esta nueva etapa del desarrollo, recordando la función del campo de fuerzas como medio transmisor de acciones eléctricas y magnéticas.

La teoría que propongo puede, por tanto, llamarse una teoría del campo Electromagnético, puesto que tiene que ver con el espacio en la vecindad de los cuerpos eléctricos y magnéticos, y puede llamarse teoría Dinámica, porque se supone que 
en ese espacio hay materia en movimiento, por la cual se producen los fenómenos electromagnéticos observados ${ }^{46}$.

La naturaleza material del medio transmisor pasa a primer plano del desarrollo. Se trata de un tipo de sustancia que llena el espacio. De sus características, Maxwell excluye la que denomina "materia pesada" [gross matter] por su incapacidad de transmitir la luz y el calor. Por el contrario, ese medio "etéreo" debe ser capaz de traspasar los cuerpos y está dotado de movimiento. Constituye, pues, un continuo interconectado (en este aspecto sigue siendo válido el modelo hidrodinámico). De forma análoga a lo que ocurre en la materia fluida, el desplazamiento de la sustancia circunscrita a una región determinada depende del desplazamiento del resto. En virtud de las propiedades elásticas, los movimientos que se producen en unas regiones del conjunto se trasfieren a otras regiones. En consecuencia, no se producen interacciones instantáneas entre las regiones del espacio y todo movimiento de masa fluida lleva asociado una cierta trasferencia de energía.

El medio es por tanto capaz de recibir y almacenar dos tipos de energía, esto es, energía "actuante" dependiente del movimiento de las partes y energía "potencial"

46 Maxwell, J. C., The Scientific Papers of James Clerk Maxwell, Dover Publications, 2003, vol. 1: 527 . 
Naturaleza y Libertad. Revista de estudios interdisciplinares. Número 1, 2012. ISSN: 2254-9668

dependiente del trabajo que el medio hará para recobrarse del desplazamiento en virtud de su elasticidad ${ }^{47}$.

Por consiguiente, el modelo del fluido es el resultado de una idealización obtenida a partir de un fluido real en movimiento, en el que unas zonas se mueven con distinta velocidad respecto a otras, pero sin romper la continuidad espacial. Cualquier porción de la masa fluida trasporta energía debido a su velocidad, denominada "energía cinética" (o bien, "energía actuante" según el término usado por Maxwell). Así mismo, cualquier región que, en un momento dado, se deforma por efecto de las presiones de otra región contigua tenderá a recuperar su forma, tal como ocurre con un sólido elástico que sufre deformaciones debido a la presión exterior. Esta última clase de energía asociada con el movimiento interno del medio, recibe el nombre de "energía potencial". En particular, cuando se propaga una onda en el interior de un medio que tiene las características que estamos describiendo, se produce una transformación de una de las formas de energía en la otra. Es decir, tiene lugar una transferencia mutua en el seno de la masa fluida, entre la energía asociada al movimiento de unas regiones del fluido y la energía elástica asociada a la recuperación de la forma geométrica de otras regiones.

Importa señalar que la energía es una magnitud física derivada que se define a partir de otras magnitudes, tales como la masa y la veloci-

47 Maxwell, J. C., The Scientific Papers of James Clerk Maxwell, Dover Publications, 2003, vol. 1: 528 . 
dad (en el caso de la energía cinética). Estas últimas guardan una relación más directa con el objeto físico considerado. No así, la energía cuya definición se hace en función de otras magnitudes, siendo, por tanto, un concepto que está más alejado de las propiedades sensibles de la materia. Por esto mismo, por su carácter más abstracto, resulta más adecuado para expresarlo mediante el lenguaje matemático.

A partir de aquí, sin abandonar el modelo hidrodinámico, las nuevas magnitudes energéticas referidas al fluido ideal en movimiento, pasarán a un primer plano del desarrollo hasta llegar —en la Parte III del artículo que comentamos- a la formulación de las ecuaciones del campo electromagnético. Este nuevo rumbo que toma la investigación del científico escocés se realiza sin abandonar los logros anteriores; más bien, se trata de otro modo de acometer la construcción de la teoría mediante el cual conseguirá integrar los resultados ya obtenidos y proporcionar al mismo tiempo una explicación del fenómeno de propagación de la luz.

Recordemos brevemente el camino recorrido desde la primera versión del modelo, cuando Maxwell comienza imaginando un fluido imponderable e incompresible, cuyas partículas siguen "líneas de corriente”. En el segundo artículo, las partículas del fluido están dotadas de masa y existen presiones internas variables que originan rotaciones o vórtices. Finalmente, en el tercer artículo se consideran los intercambios energéticos que se producen en la masa fluida en movimiento que, en lo sucesivo, se convierten en la clave del desarrollo operativo. De este modo, Maxwell consigue una ventaja metodológica respecto a 
las versiones anteriores, al utilizar el lenguaje matemático más flexible que ha tomado de la Mecánica Analítica, basado en las llamadas coordenadas generalizadas. Desde el punto de vista epistemológico se produce un mayor alejamiento de las imágenes sensibles, debido a un proceso de creciente idealización. El resultado final es un modelo de campo electromagnético concebido como un medio continuo formado por puntos dotados de las propiedades físicas necesarias para hacer posible la transmisión de fuerzas eléctricas y magnéticas.

\subsection{PROPAGACIÓN DE LA LUZ COMO ONDA}

\section{ELECTROMAGNÉTICA}

En la parte VI de A Dynamical theory of the electromagnetic field, Maxwell presenta una prueba que corrobora la teoría obtenida. La prueba consiste en explicar la propagación de la luz mediante una nueva analogía, en este caso, basada en la propagación de ondas mecánicas en medios materiales, tales como las vibraciones sonoras que se extienden en el espacio progresando según un movimiento ondulatorio. En virtud de esta semejanza formal, Maxwell expresa la velocidad de propagación de la luz en función de dos magnitudes electromagnéticas, obtenidas por trasferencia formal de las magnitudes mecánicas ${ }^{48}$. Dicha velocidad derivada por analogía conduce a un valor numérico que concuerda con el valor experimental obtenido por Fizeau en 1894.

48 Maxwell, J. C., The Scientific Papers of James Clerk Maxwell, Dover Publications, 2003, vol. 1: 499. 
Naturaleza y Libertad. Revista de estudios interdisciplinares. Número 1, 2012. ISSN: 2254-9668

Pero además de esta comprobación empírica, la teoría de Maxwell pone de manifiesto la naturaleza electromagnética de la luz. Ya que la ecuación en derivadas parciales que rige la propagación de las ondas luminosas se deduce de las ecuaciones diferenciales del campo electromagnético. Por consiguiente, estos dos resultados corroboran la validez de la teoría del campo electromagnético. Así lo recogen las siguientes afirmaciones de Maxwell:

La concordancia entre los resultados parece mostrar que la luz y el magnetismo son afecciones de la misma sustancia y que la luz es una perturbación propagada a través del campo de acuerdo con las leyes electromagnéticas ${ }^{49}$.

A las dos comprobaciones ya señaladas, algunos años después, se sumó una tercera y definitiva aportada por Heinrich Hertz (18571894), cuando utilizó circuitos eléctricos oscilantes y consiguió por primera vez emitir ondas electromagnéticas.

\section{INSTRUMENTALISMO Y REALISMO EN LA TEORÍA ELECTROMAGNÉTICA}

En resumen, el proceso constructivo de la teoría del campo electromagnético ha puesto de manifiesto su carácter instrumental y operativo. Maxwell consigue el objetivo de dar unidad a los diversos 49 Maxwell, J. C., The Scientific Papers of James Clerk Maxwell, Dover Publications, 2003, vol. 1: 580 . 
fenómenos eléctricos y magnéticos que no contaban con una explicación unitaria coherente. El método seguido para lograrlo ha sido recurrir a la construcción de analogías, a partir de varias teorías mecánicas, tales como la mecánica de fluidos, la elasticidad y la mecánica analítica. No se ha pronunciado sobre la naturaleza o estructura interna de la electricidad y el magnetismo. No ha hecho hipótesis sobre el origen de las propiedades magnéticas o eléctricas de los materiales correspondientes. La tarea de Maxwell se ha centrado únicamente en los hechos experimentales probados, tales como la atracción y repulsión de cuerpos cargados o la trasmisión de electricidad a lo largo de un conductor, o bien, en los efectos magnéticos producidos por una corriente eléctrica. Ha prescindido de las otras propiedades de la materia y asimismo ha eliminado las características particulares de los cuerpos. De este modo de proceder, se derivan algunas consecuencias que vamos a detallar a continuación.

Con relación a los conceptos que forman parte de la teoría, cabe afirmar que no son equiparables a los utilizados en el lenguaje común para referirse a los objetos materiales. Los términos científicos han sido construidos con la finalidad de ser útiles y operativos en la descripción de la teoría, pero no designan objetos materiales identificables. Cada término científico no tiene su referente sensible en la realidad material. Así, por ejemplo, la noción de "línea magnética", que juega un papel central en la teoría, no tiene existencia material, sino que, ha surgido como una idealización cuya descripción geométrica se debe a Faraday y acaba adoptando la forma de la ecuación 
matemática que Maxwell establece. Por el contrario, en el lenguaje ordinario, una expresión tal como, "una corriente de agua" designa una realidad sensible perfectamente identificable. En este caso, el nombre con el que se designa a ese objeto material procede de la observación sensible, a diferencia del significado de los términos científicos que no son objetos identificables por los sentidos ${ }^{50}$.

Maxwell ha utilizado las nociones y leyes mecánicas en su aspecto puramente formal, como soporte sensible de la imaginación, extrayendo su dimensión estrictamente relacional y prescindiendo del significado mecánico. Ha vaciado de contenido los términos hidromecánicos, como por ejemplo, "presión” o "masa”, "línea de corriente", etc. El resultado obtenido es una estructura lingüística precisa, coherente, con una función descriptiva, en la que los términos tienen un carácter simbólico y siguen criterios sintácticos, no semánticos. En consecuencia, el tipo de teoría construida debe encuadrarse dentro de un criterio pragmático y operativo, como un instrumento de cálculo.

50 Las sensaciones, como tales, no constituyen los referentes semánticos de los términos científicos. El método aplicado por Maxwell para la construcción de su teoría indica que en la definición de los términos científicos no intervienen las impresiones sensoriales, sino que responde a una libre determinación realizada en consonancia con la observación experimental. En el lenguaje científico, los términos o magnitudes son signos que no representan entes materiales. Así, la "carga eléctrica” o el "potencial" no son observables, aunque sí lo sean sus efectos externos (el movimiento de un cuerpo cargado impulsado por una fuerza eléctrica). Por el contrario, en el lenguaje común, la palabra "árbol", por ejemplo, designa un objeto real percibido por los sentidos. 
Por todo ello, considerando sólo el proceso de construcción de la teoría del campo electromagnético, cabría admitir el papel instrumental de la ciencia, entendida como un lenguaje o formulación simbólica, sin la pretensión metafísica de llegar al conocimiento de naturalezas o esencias. A este respecto Ana Rioja ha escrito:

No se precisa salir del ámbito de los fenómenos como símbolos para acceder a la naturaleza última y esencial de lo que se nos aparece. La ciencia ni es ni pretende ser un conocimiento de esencias o de causas últimas, pero ello no quiere decir que no sea conocimiento en absoluto ${ }^{51}$ [Cursiva añadida].

Los términos utilizados en el lenguaje científico poseen su significado principalmente por la relación contextual que adquieren dentro de la teoría, pero no se refieren de modo directo a los objetos materiales tal y como se perciben por los sentidos, sino a los objetos idealizados del modelo. Si bien, en las teorías más abstractas las magnitudes que se utilizan son símbolos no figurativos. Así ocurre, por ejemplo, con el término científico "energía" que admite varias formas (cinética, potencial, térmica, etc.). Por ejemplo, la energía cinética de un cuerpo material de forma esférica hace referencia a la propiedad física que tiene ese objeto material debido al movimiento, pero no admite una representación sensible, como sucede por el contrario con el propio objeto esférico.

51 Rioja, A., "La filosofía de la ciencia de G. Berkeley". La ciencia de los filósofos. Thémata, 1996, n. 17: 164. 
El procedimiento analógico aplicado por Maxwell y la construcción de la teoría avalan la tesis de Berkeley sobre la ciencia, como gramática de la Naturaleza. La tarea del científico es construir una estructura gramatical ordenada, bajo la condición imprescindible de que las reglas sintácticas sean aplicables y por tanto contrastables experimentalmente. En este sentido, la teoría debe ser operativa, es decir, que las magnitudes definidas puedan ser cuantificables, directa o indirectamente, mediante determinadas operaciones de medida. Mediante las medidas experimentales de algunas magnitudes, toda la estructura simbólica en conjunto adquiere significado, gracias a la cohesión lógica interna. Esto es así, aunque algunos de los símbolos no admitan una representación sensible e incluso a pesar de que esas representaciones carezcan de sentido en el lenguaje común, como ocurre, por ejemplo, con los modelos utilizados en la mecánica cuántica o en la física del átomo.

En este sentido, la aplicación de la Dinámica Analítica llevada a cabo por Maxwell en el tercer artículo ya citado, es un paso decisivo hacia la construcción de esa estructura formal y simbólica. La aportación más notable consiste en su capacidad de enlazar por vía deductiva, empleando métodos matemáticos, los datos experimentales de partida con las consecuencias verificables de la teoría. Por esto, para Maxwell el valor principal del método analítico iniciado por Lagrange reside en 
su precisa capacidad deductiva, partiendo de los datos observables ${ }^{52}$. Este camino lógico exige restringir el significado mecánico, ya que el tránsito entre los datos medidos y los resultados obtenidos debe hacerse por deducción matemática.

Maxwell admite el carácter operacional de la teoría; así se desprende de sus comentarios a los trabajos publicados en 1867 por sus colegas W. Thomson y P. Tait. La función del cálculo matemático es establecer una conexión lógica entre los datos de entrada y los valores que se obtienen en la observación del fenómeno. Para explicar su funcionamiento no es necesario conocer con detalle los procesos internos de un complejo mecanismo ${ }^{53}$.

52 Chalmers, A. F., "Maxwell's methodology and his applications of it to electromagnetism”. Studies in History and Philosoby of Science. 1973, vol. 4, no. 2: 156. 53 Maxwell se refiere al método de Lagrange con una breve ilustración sobre el funcionamiento de un sencillo mecanismo de uso doméstico (belfry) consistente en una serie de campanillas accionadas mediante un sistema de cuerdas y poleas. Sin necesidad de conocer con detalle el mecanismo, con las posiciones y velocidades de las cuerdas será posible determinar la energía potencial y cinética, aplicando las ecuaciones de Lagrange. Por esto, manteniendo los datos de entrada y de salida, es posible suponer una variedad de mecanismos intermedios que servirían para explicar el sistema en su conjunto. El método lagrangiano justificaría un enfoque instrumentalista de las teorías, entendidas como un lenguaje simbólico que establece relaciones matemáticas entre los datos experimentales de partida y los resultados obtenidos, sin aportar conocimiento real ("Thomson and Tait's Natural Philosophy", en: Maxwell, J. C., The Scientific Papers of James Clerk Maxwell, Dover Publications, 2003, vol. 2: 783). 
No obstante al carácter instrumental de la teoría del campo electromagnético, puesto en evidencia por su proceso de construcción, Maxwell parece inclinarse por rechazarlo. Sus comentarios recogidos en la disertación sobre la transmisión de fuerzas en el espacio, titulada On Action at a Distance y publicada en Proccedings of the Royal Institution of Great Britain son muy elocuentes. En esa ocasión, describiendo la propagación de la luz en el espacio interestelar mediante las líneas magnéticas que lo recorren, hace referencia a tales líneas en términos que podrían calificarse de realistas.

Y estas líneas no deben ser consideradas como meras abstracciones matemáticas. Son las direcciones en las que el medio está ejerciendo una tensión como la que ejerce una cuerda, o más bien, como la que realizan nuestros músculos ${ }^{54}$.

La anterior afirmación de Maxwell sugiere que el modelo idealizado, a pesar de ser concebido como un instrumento constructivo, opera con tal convicción psicológica en la mente de su autor que le lleva a adjudicar existencia real al campo electromagnético, con propiedades similares a las de un medio elástico sometido a tensiones y capaz de propagar ondas luminosas, como lo hace el aire o el agua con las ondas acústicas.

Pero, de hecho no queda justificado el salto desde la construcción formal de la teoría del campo electromagnético a la interpretación 54 Maxwell, J. C., The Scientific Papers of James Clerk Maxwell, Dover Publications, 2003, vol. 2: 323 . 
realista, que cabe deducir del comentario anterior de Maxwell negando el carácter abstracto (simbólico) de las líneas magnéticas. Y, más aún, teniendo en cuenta que, en el proceso de construcción de la teoría, dichas líneas han sido introducidas en el modelo como idealizaciones, a partir de la disposición adoptada por pequeñas porciones de hierro en presencia de un imán magnético. Por consiguiente y en consonancia, con el método constructivo del campo electromagnético no parece admisible tal existencia real del campo electromagnético. Faraday y Maxwell fracasaron ante el reto de determinar su consistencia física, sin embargo, desde el punto de vista epistemológico, la valiosa aportación de ambos radica, ante todo, en el modelo del campo de fuerzas y no en el descubrimiento de la hipotética realidad material que investigaban.

En este sentido y refiriéndose al trabajo de Maxwell, P. M. Harman destaca la teoría de las "líneas de fuerza" "como representación geométrica de la distribución espacial de la fuerza en el campo, más bien que como una representación fisica del campo" ${ }^{55}$. Y, de hecho, la noción de campo de fuerzas va perdiendo paulatinamente su interés como entidad física real, al tiempo que gana terreno como representación simbólica, ante la necesidad de describir con mayor unidad conceptual los diferentes fenómenos electromagnéticos. Es decir, su estructura se concibe como asiento de propiedades electromagnéticas y

55 Harman, P. M., Energy, Force, and Matter. The conceptual Development of Nineteenth-Century Physics, Cambridge, University Press, 1982: 88 [Cursiva añadida]. 
en cuanto trasmisor de esas interacciones, incluidas las ondas luminosas. Por tanto, hemos de concluir que la evolución conceptual del modelo de campo electromagnético evidencia una progresiva idealización o "desmaterialización", hasta jugar el papel de un símbolo dentro del lenguaje científico.

De todos modos, el descubrimiento de posibles realidades físicas ha ejercido siempre un gran atractivo entre los científicos que siguieron los pasos de Maxwell, e impulsados por ese objetivo, trataron de determinar la naturaleza del campo electromagnético. La comprobación experimental de la teoría electromagnética les animó a continuar indagando acerca de la constitución material de esa hipotética sustancia, que recibió el nombre de éter. Así, para el científico holandés Hendrik A. Lorentz (1853-1928), el éter era un medio rígido, no mecánico, donde se producían interacciones, tanto entre las cargas eléctricas, como entre esas partículas y el propio éter. Posteriormente, Einstein conferirá al campo electromagnético una compleja estructura matemática en la que adquieren unidad formal los fenómenos gravitatorios y electromagnéticos, quedando ambos incluidos en un mismo marco espacio-temporal. Pero, esa mayor cohesión formal entre sucesos y entidades físicas tan dispares obligaba a alejarse de la existencia material del éter. A esta búsqueda infructuosa se refiere Einstein en términos elocuentes: 
Naturaleza y Libertad. Revista de estudios interdisciplinares. Número 1, 2012. ISSN: 2254-9668

[...] el problema de idear un modelo mecánico del éter se hacía cada vez menos interesante y resultó ser cada vez más descorazonador, a causa del carácter artificial $\mathrm{y}$ forzado de las suposiciones ${ }^{56}$.

Efectivamente, el éter escapaba no sólo al intento de clasificarlo como realidad mecánica sino a toda pretensión de detectar su existencia. Las palabras de Einstein no dejan lugar a duda: "han fallado todos los intentos de convertir el éter en una realidad"57. Así pues, ante tal resultado, a juicio del científico alemán, lo mejor era ignorarlo completamente y "tratar de no mencionar más su nombre", admitiendo sencillamente que "nuestro espacio tiene la propiedad física de trasmitir las ondas electromagnéticas". Esta malograda aventura científica produjo, no obstante, un gran valor metodológico al mostrar por vía empírica que no era posible encontrar una supuesta realidad física. Puesto que, al fin y al cabo, tal pretendida realidad estaba sustentada en una ficción, esto es, en la construcción de un modelo idealizado o esquema conceptual utilizado como soporte sensible al pensamiento para elaborar la teoría.

La conclusión que cabe extraer de las consideraciones anteriores en torno al método analógico de Maxwell apunta hacia el carácter instrumental de la ciencia. Conclusión avalada por la construcción de la

56 Einstein, A.; Infeld, L., The Evolution of Physics, Cambridge. University Press, 1961: 153.

57 Einstein, A.; Infeld, L., The Evolution of Physics, Cambridge. University Press, 1961: 175 . 
teoría campo electromagnético, prescindiendo de la naturaleza física de los fenómenos observados. De acuerdo con M. Morrison, admitimos que el método analógico seguido por Maxwell le permite soslayar el problema de la constitución sustancial o realidad física de los fenómenos que estudia. Al hacerlo así, excluye las hipótesis existentes sobre la naturaleza del medio. Por eso, "los modelos y conceptos [que utiliza] han de ser entendidos como imágenes constructivas que representan posibilidades físicas o que han de ser empleadas con meros propósitos heurísticos" ${ }^{78}$. Se demuestra que la elección de Maxwell fue acertada al rechazar hipótesis de partida que le habrían limitado el desarrollo posterior.

En consecuencia, el procedimiento utilizado para obtener la teoría del campo electromagnético habla a favor de una ciencia física de carácter instrumental que no necesita fundamentar sus principios teóricos, ni sus deducciones en el conocimiento de entidades reales. Le basta la libre construcción creativa de modelos idealizados y de objetos abstractos de carácter simbólico definidos (no deducidos, ni inducidos) en consonancia con la observación experimental de los fenómenos.

Pese a todo, es obligado preguntarse si el resultado obtenido, además de proporcionar un lenguaje operativo útil para describir los fenómenos, no es también capaz de aportar algún tipo de conocimiento sobre la realidad del mundo material. Es decir, cabe electromagnetic theory". 1992, p. 144. 
preguntarse si el lenguaje de la ciencia no encierra algún tipo de conocimiento. Puesto que la teoría ha de someterse a comprobación experimental, pensamos que la cuestión planteada admite una respuesta afirmativa. No, en virtud de un realismo ingenuo que tomase los modelos y los conceptos definidos como representaciones literales de objetos reales, sino porque las leyes de la teoría son relaciones estables entre entidades construidas que traducen al lenguaje teórico los nexos permanentes existentes entre los objetos materiales. Así, por ejemplo, la ley de la gravitación relaciona las masas y la distancia entre cuerpos materiales, dando a conocer mediante el lenguaje simbólico, un rasgo propio y permanente del mundo físico. La ley de la gravitación puede ser expresada de forma diferente de acuerdo con el marco teórico que se utilice, sea éste newtoniano o einsteniano, pero, en uno o en otro, se refiere al mismo previsible modo de proceder que tienen los cuerpos naturales. Lo dicho es aplicable a las leyes que rigen los fenómenos electromagnéticos o cualquier otro fenómeno. Por esto, cabe afirmar que las teorías físicas captan — sin agotar su posible conocimiento- determinados aspectos de la naturaleza, no porque descubran o desvelen entidades o sustancias, sino porque expresan, con ayuda del lenguaje matemático, interacciones naturales observables, fijas y bien establecidas ${ }^{59}$.

59 Con este mismo sentido, P. Duhem ha destacado el papel de las teoría científica en los siguientes términos "Así, la teoría física [...] nunca revela realidades escondidas bajo apariencias sensibles; pero cuanto más completa llega a ser, más captamos que el 
La estructura formal simbólica y coherente de la teoría tiene sentido en cuanto conjunto indiviso y adquiere significado físico por vía experimental. De este modo, la teoría científica, a pesar de su carácter simbólico, proporciona un conocimiento parcial de las interacciones que se dan en la naturaleza. Las teorías captan los fenómenos naturales con un lenguaje metafórico, en cierto modo. Por esto, no es aplicable a las teorías, el calificativo de "Velo de las Palabras" con el que Berkeley se refiere a los términos científicos, los cuales — según él— por carecer de significación no desvelan la realidad, sino que la ocultan. Por el contrario, las teorías científicas dejan vislumbrar algunos rasgos del mundo físico, sirviéndose del lenguaje simbólico.

Miguel Ángel Herrero Universidad Politécnica de Madrid ETSI Telecomunicación Ciudad Universitaria s/n. 28034 Madrid maherrer@fis.upm.e

orden lógico en que la teoría organiza las leyes experimentales es el reflejo de un orden ontológico y más sospechamos que las relaciones que establece entre los datos observados corresponden a relaciones reales entre las cosas" (Duhem, P., The Origin of Statics, Kluwer, 1991: 26-27). 